\title{
Interação entre Formigas e Aleirodídeos (Sternorrhyncha, Aleyrodidae) em Cultivo Orgânico de Tangerina cv. Poncã (Citrus reticulata Blanco)
}

\author{
William Costa Rodrigues ${ }^{\circledR} \bowtie$ Paulo Cesar Rodrigues Cassino ${ }^{2}$
}

1. Universidade Severino Sombra, e-mail: wcrodrigues@ebras.bio.br (Autor para correspondência ${ }^{\bowtie}$ ). 2. Universidade Federal Rural do Rio de Janeiro/Universidade Severino Sombra, e-mail: pr.cassino@uol.com.br.

\author{
EntomoBrasilis 4 (3): 119-124 (2011)
}

\begin{abstract}
Resumo. Os aleirodídeos são atendidos por formicídeos, conferindo assim proteção e dispersão, o que dificulta o controle destes sugadores. A interação Formicidae-Sternorrhyncha ocorre, principalmente, devido à produção de uma substância açucarada denominada honeydew. O objetivo do trabalho foi realizar o levantamento, estudo da diversidade, frequência, constância e dominância das espécies de formigas atendente de aleirodídeos no período noturno e diurno. Foram realizados levantamentos diurnos e noturnos durante o período de outubro de 2002 a outubro de 2003 . As espécies de aleirodídeos encontradas foram Aleurothrixus floccosus Maskell, Aleurotrachelus cruzi Cassino, Singhiella citrifolii (Morgan) e Paraleyrodes bondari Peracchi. Foram constatadas 12 espécies de formigas associadas a $A$. floccosus, quatro espécies associadas à $A$. cruzi e três espécies associadas a $P$. bondari. Não foi verificada associação de formicídeos com $S$. citrifolii. Verificou-se que a interação entre $A$. floccosus e as formigas foi mais diversa (o,743) com menor dominância (o,3194) e equitabilidade (o,6882), sendo Brachymyrmex sp a espécie mais frequente $(31,94 \%)$ e constante. Em $P$. bondari a diversidade foi 0,477 e dominância 0,3333 , sendo a equitabilidade $J$ igual a 1. Em $A$. cruzi, a diversidade foi 0,422 , a dominância 0,6098 e equitabilidade J 0,7013. Sobre esta espécie de aleirodídeo há uma dominância da formiga Crematogaster sp. 1, com frequência de 60,98\%, apesar de acessória. A estreita relação de atendimento de A. floccosus por 12 formicídeos demonstra uma preferência destes pelos excrementos (honeydew) do aleirodídeos, seja pela quantidade ou qualidade do mesmo.
\end{abstract}

Palavras-Chave: Atendimento; Citricultura; Cultivo orgânico; Formicidae; Interação formiga-aleirodídeo.

\section{Interaction between and Whiteflies (Sternorrhyncha, Aleyrodidae) in Organic Cropping of the Tangerine cv. Ponkan (Citrus reticulata Blanco)}

Abstract. The whiteflies are tending by ants, which give protection and dispersion make the control of those suckers more difficult. The interaction ant-Sternorrhyncha occurs, mainly due to the production of a sugared material named honeydew. The aim of the study as realized sampling, diversity study, frequency, constancy and dominance of the ant's whiteflies tending species in night and day period. The sampling were conducted from October 2002 to October 2003. The species of whiteflies detected were Aleurothrixus floccosus Maskell, Aleurotrachelus cruzi Cassino, Singhiella citrifolii (Morgan) and Paraleyrodes bondari Peracchi. The study concluded that 12 species of ants were associated with A. floccossus, 4 species with A. cruzi and 3 species with $P$. bondari. Associations between ants and $S$. citrifolli where not observed. Among the species of ants associated with whiteflies, those ones associated with $A$. floccosus presented the major diversity (o.743) and the minor dominance (0.3194) and equitability J (o.6882). Brachymyrmex sp. was the most frequent species (31.94\%) and also the most constant. P. bondari showed a diversity of 0.477 , the dominance of 0.3333 and the equitability $\mathrm{J}$ 1. To $A$. cruzi, the diversity was of 0.422 , dominance of 0.6098 e equitability $J$ of 0.7013 . This species of whiteflies show a dominance of the species Crematogaster sp. 1, with the frequency of $60.98 \%$ although it was secondary. The close relationship between A. floccosus and 12 ants demonstrates a preference for the excrements (honeydew) of the whiteflies, for the quantity as well as for the quality of the honeydew.

Keywords: Ant-whiteflies interaction; Citriculture; Formicidae; Organic Cropping; Tending.

A associação de formigas e aleirodídeos produtores honeydew (excreções açucaradas) são frequentes, devido formicídeos, o que beneficia os homópteros através da proteção. Entretanto, esta proteção não é absoluta, tendo em vista que os predadores e parasitóides desenvolvem estratégias sofisticadas para evitar a proteção das formigas (WAY 1963). Desta forma o resultado do mutualismo, quando existente, proporciona benefícios recíprocos obtidos pela associação entre as espécies (AdDicotT 1978).

O número de espécies de formigas coletando honeydew em um pomar cítrico é relativamente considerável (123 espécies), ocasionado desta forma outbreaks de insetos prejudiciais a estes pomares (SAMWAYs et al. 1982).

As formigas através do processo de atendimento a aleirodídeos podem afetar negativamente as plantas, porque estes últimos extraem grande quantidade de seiva elaborada e excretam honeydew, promovendo o desenvolvimento de fungos, propiciando a diminuição da taxa fotossintética e abscisão foliar (Queiroz \& Oliveira 2001). Mas é importante ressaltar que todos os homópteros produtores de honeydew, também são tão prejudiciais quanto os aleirodídeos, pelos mesmos motivos (RODRIGUES 2004).

$\mathrm{O}$ atendimento de aleirodídeos produtores de honeydew por formigas é quase que obrigatório, pois quase todos os Sternorrhyncha e Auchenorrhyncha necessitam eliminar o excesso de honeydew produzido por eles, entretanto muito destes são ou não atendidos, ou somente são atendidos acidentalmente por formigas (DELABIE 2001). Desta forma, eliminação física ou mecânica do honeydew é certamente um dos mais importantes problemas na evolução dos homópteros e a incapacidade de eliminá-lo pode significar o afogamento nesta substância (GULAN 
\& Kosztarab 1997).

O relacionamento de atendimento de Sternorrhyncha ou Auchenorrhyncha por formigas demonstra uma verdadeira ligação entre os dois organismos e constitui um subsequente passo na evolução destes grupos. Segundo Delabie (2001), os casos de trofobiose entre formigas-homópteros são comumente encontrados em Sternorrhyncha, possivelmente como uma consequência da incapacidade de locomoção por parte de muitos destes ou por causa da abundância e qualidade do honeydew produzido por outros homópteros (Aphididae). Entretanto este processo de atendimento também depende de outros aspectos, tais como as adaptações morfológicas, comportamentais, fisiológicas e químicas dos homópteros para associar-se com formigas, podendo o honeydew conter atrativos específicos para as formigas (BUCKLEY 1987).

O objetivo do trabalho foi realizar o levantamento, estudo da diversidade, frequência, constância e dominância das espécies de formigas atendente de aleirodídeos no período noturno e diurno em cultura de citros orgânico.

\section{MATERIAL E MÉTODOS}

O estudo foi realizado na Fazendinha Agroecológica (EMBRAPA-Agrobiologia/ UFRuralRJ/Pesagro), situada no bairro Ecologia do município de Seropédica, RJ. A Fazendinha Agroecológica é uma área experimental, onde são desenvolvidos estudos sobre Agroecologia dentro do SIPA (Sistema Integrado de Produção Agroecológica).

$\mathrm{O}$ pomar de tangerina (Citrus reticulata Blanco) $\mathrm{cv}$. Poncã, com cinco anos de plantio, contendo 30 plantas com espaçamento de $3,5 \times 3,5 \mathrm{~m}$, encontra-se em início de produção, estando circundada por plantio de graviola (Anonas muricata L.) e fruta-do-conde (Anonas squamosa L.), além de ter no seu subbosque a presença de grama batatais (Paspalum notatum Flugge). O pomar não recebeu qualquer tratamento para controle dos insetos fitoparasitos (pragas), tendo apenas recebido adubação orgânica com esterco curtido de galinha, durante o período do estudo.

O levantamento e o monitoramento para o estudo da interação das formigas com os aleirodídeos foram realizados entre outubro de 2002 e outubro de 2003, exceto no mês de janeiro 2003, perfazendo desta forma 12 meses de amostragens, baseando-se na metodologia de "presença-ausência", proposta por CASSINO \& RODRIGUES (2004), sendo verificada a atividade das formigas sobre os homópteros, ou seja, se as mesmas utilizam as excreções açucaradas destes aleirodídeos.

$\mathrm{O}$ número de plantas monitoradas foi fixo, $10 \mathrm{em}$ 30 plantas e as plantas amostradas não variaram entre os monitoramentos. Este procedimento foi realizado verificando as plantas que possuíam população de homópteros atendida por formigas. As plantas foram imaginariamente divididas em quatro quadrantes. Altura de observação foi entre 1,30 e 1,70m. A presença dos homópteros, formigas e predadores foi observada por aproximadamente cinco minutos em cada quadrante, ou seja, 20 minutos por planta, padronizando o tempo de monitoramento em cada quadrante, reduzindo assim possíveis erros. Em cada planta foram monitorados quatro quadrantes, assim em cada levantamento foram monitorados 40 quadrantes (10 plantas).

Os levantamentos diurnos foram realizados a cada 15 dias, sempre às 9:ooh e os levantamentos noturnos foram realizados mensalmente e sempre às 21:0oh. No mesmo dia que ocorria o levantamento noturno era realizado o monitoramento diurno. Neste levantamento havia uma variação no tempo do monitoramento de 5 a 7 minutos por quadrante no período noturno.

Para as análises dos dados foram utilizados os seguintes softwares: Quantitative Analysis in Ecology incluído em BROWER et al. (1997); DivEs v2.o (Rodrigues 2005) e Planilha de Cálculo do Open Office.org (Sun Microsystem 2005).

Para avaliar a diversidade, dominância, equitabilidade, constância e frequência dos formicídeos associados aos aleirodídeos foram utilizados os seguintes índices: diversidade de Shanon-Wiener, dominância de Berger-Parker, equitabilidade $\mathrm{J}$, constância (onde, espécies constantes (w): estão presentes em mais de 50\% dos levantamentos realizados; espécies acessórias (y): estão presentes entre 25 a 50\% dos levantamentos; e espécies acidentais (z): estão presentes em menos de $25 \%$ dos levantamentos) e frequência.

\section{RESULTADOS E DISCUSSÃO}

Aleirodídeos. Foi verificada a ocorrência de quatro espécies durante o período de estudo: Aleurothrixus floccosus Maskell; Aleurotrachelus cruzi Cassino; Singhiella citrifolii (Morgan) e Paraleyrodes bondari Peracchi. A ocorrência destas espécies já havia sido constatada no Estado do Rio de Janeiro em plantios comerciais de citros, com cultivo convencional (CASsino \& Nascimento 1999; Cassino et al. 2004). Para cultivo orgânico de citros, as citações estão registradas apenas em anais de congressos. Nestes estudos as espécies verificadas foram: $A$. floccosus, $A$. cruzi e $P$. bondari. Desta forma, a ocorrência de $S$. citrifolii, ainda não havia sido registrada. O relato da ocorrência destes insetos é importante, pois além de causarem danos diretos, atuam de forma indireta, podendo algumas espécies transmitir viroses às plantas cítricas (KARASEV 2000).

Formigas. As espécies de formigas associadas aos aleirodídeos foram: Formicinae - Brachymyrmex sp., Camponotus rufipes (Fabricius), Camponotus atricefs (Fabricius), Camponotus crassus (Mayr) e Camponotus sp. 1; Myrmicinae-Crematogaster sp. 1, Crematogaster sp. 2, Cephalotes pusillus (Klug), Pheidole sp. e Dorymyrmex sp.; Ponerinae - Ectatomma brunneum (Fabricius); Pseudomyrmecinae - Pseudomyrmex termitarius (Fr. Smith). Nas subfamílias Myrmicinae e Formicinae verificouse cinco espécies, equivalendo a 41,67\% das formigas encontradas; as duas outras subfamílias equivaleram a $8,33 \%$. Resultados semelhantes foram encontrados por Castro et al. (1989), onde a subfamília Myrmicinae foi mais abundante, com 50\% e Formicinae representou $22 \%$ das morfoespécies encontradas. O gênero com maior frequência foi Camponotus, com 57,14\% dos gêneros encontrados (sete), pois as espécies pertencentes à Formicinae estão mais adaptadas à alimentação líquida (FowLER et al. 1991).

As espécies encontradas no pomar necessariamente estavam associadas à pelo menos uma espécie de homóptero, configurando a interação de atendimento. $\mathrm{O}$ maior número de indivíduos verificados atendendo A. floccosus foi Brachymyrmex sp. (291), Crematogaster sp. 1 (246), Camponotus sp. (165) e C. rufipes (106). As espécies com menor número de indivíduos foram: E. brunneum (2), Crematogaster sp. 2 (4) e Dorymyrmex sp. (5). Espécies pertencentes à subfamília Myrmicinae e Formiciane, verificando maior abundância destas subfamílias, corroborando CASTRO et al. (1989).

Nas subfamílias Dolicoderinae, Fromicinae e Myrmicinae, estão as formigas comumente associadas ao atendimento (CARRoll \& JANZEN 1973; HöLLDOBER \& WiLSON 1990), podendo haver também forrageamento por Pseudomyrmicinae ou Ponerinae (Kelin et al. 1992). Estes fatos confirmam os dados encontrados no presente trabalho.

No período de estudo foi verificada a proteção dos homópteros atendidos contra predadores e mesmo uma dispersão entre as plantas de um pomar, fato também verificado por WAY (1963) e SAMWAYS (1983).

Interação Formigas x Homópteros. Dentre os aleirodídeos estudados, somente $S$. citrifolii, não foi observado em associação com formigas, entretanto, a espécie $A$. floccosus foi a que se verificou maior associação (12 no período diurno e sete no período noturno). Esta associação pode ter ocorrido devido à percentagem média de infestação deste aleirodídeo $(74,6 \%)$ (Figura 1), constituindo assim fonte de recurso previsível a ser explorado por formicídeos (Fowler et al. 1991), já que a exudação 


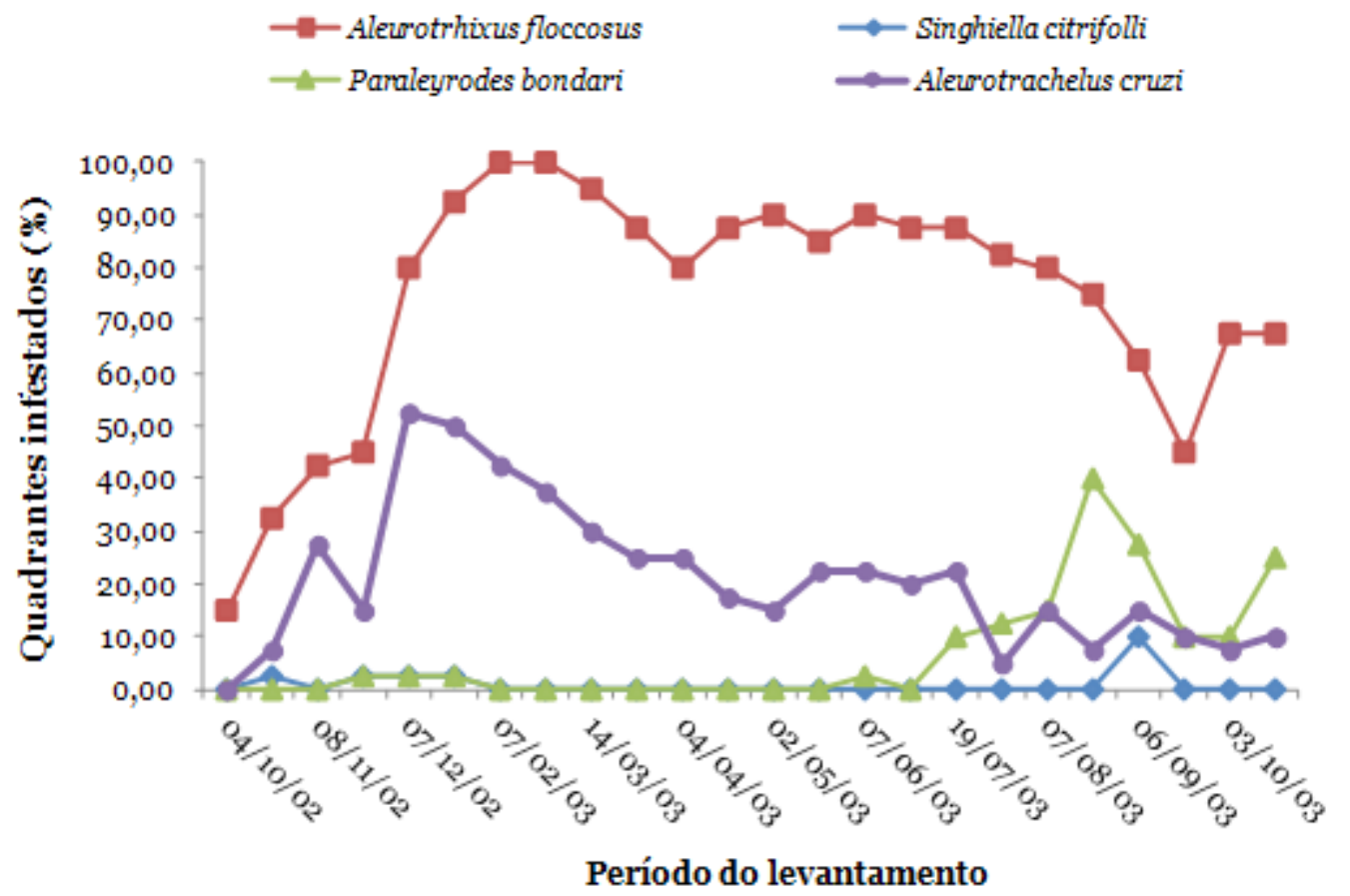

Figura 1. Percentagem de quadrantes infestados de aleirodídeos (Homoptera: Aleyrodidae), associados à tangerina cv Poncã, cultivada em sistema orgânico, no período diurno. Seropédica, RJ, Fazendinha Agroecológica. Outubro/2002 - Outubro/2003.

de honeydew deste homóptero é constante. O número de espécies de formigas associadas à $A$. cruzi no período diurno foi quatro e no período noturno duas, onde a percentagem média de infestação deste aleirodídeo foi representativa (20,94\%). Associado a $P$. bondari, observaram-se no período diurno dois indivíduos e no período noturno um, somando assim três espécies (Tabela 1).

$\mathrm{O}$ forrageamento de honeydew por formicídeo tem sido apontado como oportunista (OlIVEIRA \& BRANDÃo 1991), entretanto constitui uma fonte de alimento importante para a manutenção, principalmente pelo fornecimento de açúcares e aminoácidos (HENDRIX et al. 1999).

Em se tratando dos períodos de forrageamento, nota-se um maior número de espécies forrageando durante o dia e um menor à noite (Tabela 1). Desta forma, o hábito de forrageamento podeinfluenciar na preferência do formicídeo por um determinado período, entretanto o atendimento pode estender-se por 24 horas (Del-Claro \& Oliveira 1996).

Diversidade de Espécies, Dominância e Equitabilidade J de Formigas. Dentre as espécies de aleirodídeos, $A$. floccosus destacou-se das demais com o atendimento por 12 formigas, sendo também a espécie com maior número de formigas associadas dentre os homópteros verificados. Fato associado à disponibilidade de honeydew produzido por esta espécie ou pela qualidade deste, pois adaptações morfológicas, comportamentais, fisiológicas e químicas dos homópteros para associarem-se com formigas são aspectos importantes para que estes aleirodídeos sejam atendidos, podendo também o honeydew conter atrativos específicos para as formigas (BUCKLEY 1987; HENDRIX et al. 1999). O índice de Shanon-Wiener mostrou que A. floccosus é a espécie com maior diversidade de formigas atendentes (o,7430). Brower et al. (1997), sugere que o índice ShanonWiener (H') é mais apropriado para amostragens aleatórias de grandes comunidades ou sub-comunidades, como é o caso da comunidade estudada. A segunda espécie na classificação pelo índice de diversidade foi $P$. bondari, na qual se verificou um valor de $\mathrm{H}^{\prime}=0,4770$ e, a terceira, $A$. cruzi com valor de $\mathrm{H}^{\prime}=0,4220$. Isto indica que $A$. floccosus tem maior atendimento, devido a grande quantidade ou qualidade de honeydew produzida, em comparação com as demais espécies, pois esta composição sofre alteração de acordo com a espécie (HENDRIX et al. 1999; FISCHER et al. 2002).

Em $P$. bondari não foi verificada tendência à dominância ( $d=0,3333)$, pois somente três indivíduos, de três espécies de

Tabela 1. Associação entre formicídeos e Aleirodídeos sobre plantios de tangerina (Citrus reticulata) cv. Poncã, no período diurno (levantamentos quinzenais) e noturno. Seropédica, RJ, Fazendinha Agroecológica. Outubro/2002 - Outubro/ 2003.

\begin{tabular}{|c|c|c|c|c|c|c|c|c|}
\hline \multirow{3}{*}{ Formicídeo } & \multicolumn{8}{|c|}{ Aleirodídeo } \\
\hline & \multicolumn{2}{|c|}{ A.flocossus } & \multicolumn{2}{|c|}{ S. citrifolii } & \multicolumn{2}{|c|}{ P. bondari } & \multicolumn{2}{|c|}{ A. cruzi } \\
\hline & D & $\mathbf{N}$ & $\mathbf{D}$ & $\mathbf{N}$ & D & $\mathbf{N}$ & $\mathbf{D}$ & $\mathbf{N}$ \\
\hline Brachymyrmex sp. & + & + & - & - & - & - & - & + \\
\hline C. rufipes & + & + & - & - & + & - & + & - \\
\hline C. atricefs & + & + & - & - & - & + & + & - \\
\hline C. crassus & + & - & - & - & - & - & + & - \\
\hline Camponotus sp. 1 & + & + & - & - & - & - & + & + \\
\hline Crematogaster sp. 1 & + & + & - & - & - & - & - & - \\
\hline Crematogaster sp. 2 & + & + & - & - & - & - & - & - \\
\hline C. pusillus & + & - & - & - & + & - & - & - \\
\hline E. brunneum & + & - & - & - & - & - & - & - \\
\hline Pheidole sp. & + & - & - & - & - & - & - & - \\
\hline Dorymyrmex sp. & + & - & - & - & - & - & - & - \\
\hline P. termitarius & + & + & - & - & - & - & - & - \\
\hline Total & 12 & 7 & $\mathbf{0}$ & $\mathbf{0}$ & 2 & $\mathbf{1}$ & 4 & 2 \\
\hline
\end{tabular}

+: associação verificada; -: associação não verificada; $\mathrm{D}$ - período diurno; $\mathrm{N}$ - período noturno. 
formigas foram observados atendendo este homóptero. Assim, há um equilíbrio no número de indivíduos entre as espécies, apesar de ter havido maior diversidade em relação a $A$. cruzi.

Quando avaliada a dominância das formigas atendentes de $A$. cruzi, verificou-se valor igual a o,6098, o que significa que a espécie tem o atendimento dominante por Crematogaster sp 1, que é também a espécie de formiga mais frequente.

Quando comparada a equitabilidade (J) dos formicídeos associados aos aleirodídeos, verificou-se que há nitidamente uma maior distribuição do número de indivíduos por espécie para $P$. bondari e uma menor equitabilidade para $A$. floccosus, o que se pode entender como uma tendência à dominância de uma determinada espécie, mesmo que o índice de Berger-Parker não demonstre; ou seja, Brachymyrmex sp. e Crematogaster sp. tendem a dominar o forrageamento em A. floccosus devido ao número de indivíduos deste formicídeos associados ao aleirodídeo, 291 e 246, respectivamente.

A diversidade de Shanon-Wiener é inversamente proporcional a dominância de Berger-Parker, que pode ser verificado em $A$. floccosus (Figura 2), pois entende-se que uma maior diversidade suprimirá a dominância de uma ou mais espécies de formicídeos e vice-versa, sendo estes índices indicados para avaliação desses dois parâmetros em populações que apresentem regularidade de frequência e que não possuam valor zero frequentes nos índices, como em A. cruzi (Figura 3).

Existem casos em que as fontes alimentares encontradas são muito grandes, para uma única operária explorá-la, ocorrendo o recrutamento (Fowler et al. 1991). Assim, espécies com menor tamanho (Crematogaster spp.- 2,5 a 4,0 mm e Brachymyrmex spp. - até $3 \mathrm{~mm}$ ) necessitam de um maior número de indivíduos para forragear uma colônia de homópteros; como verificado no forrageamento de Brachymyrmex sp. (10 a 30 em média) e Crematogaster sp. 1 (10 a 20 indivíduos). Estes sistemas de recrutamento podem ser também combinados com trabalhos coordenados entre operárias para explorar recursos maiores (Fowler 1988), como foi verificado em Crematogaster sp 1

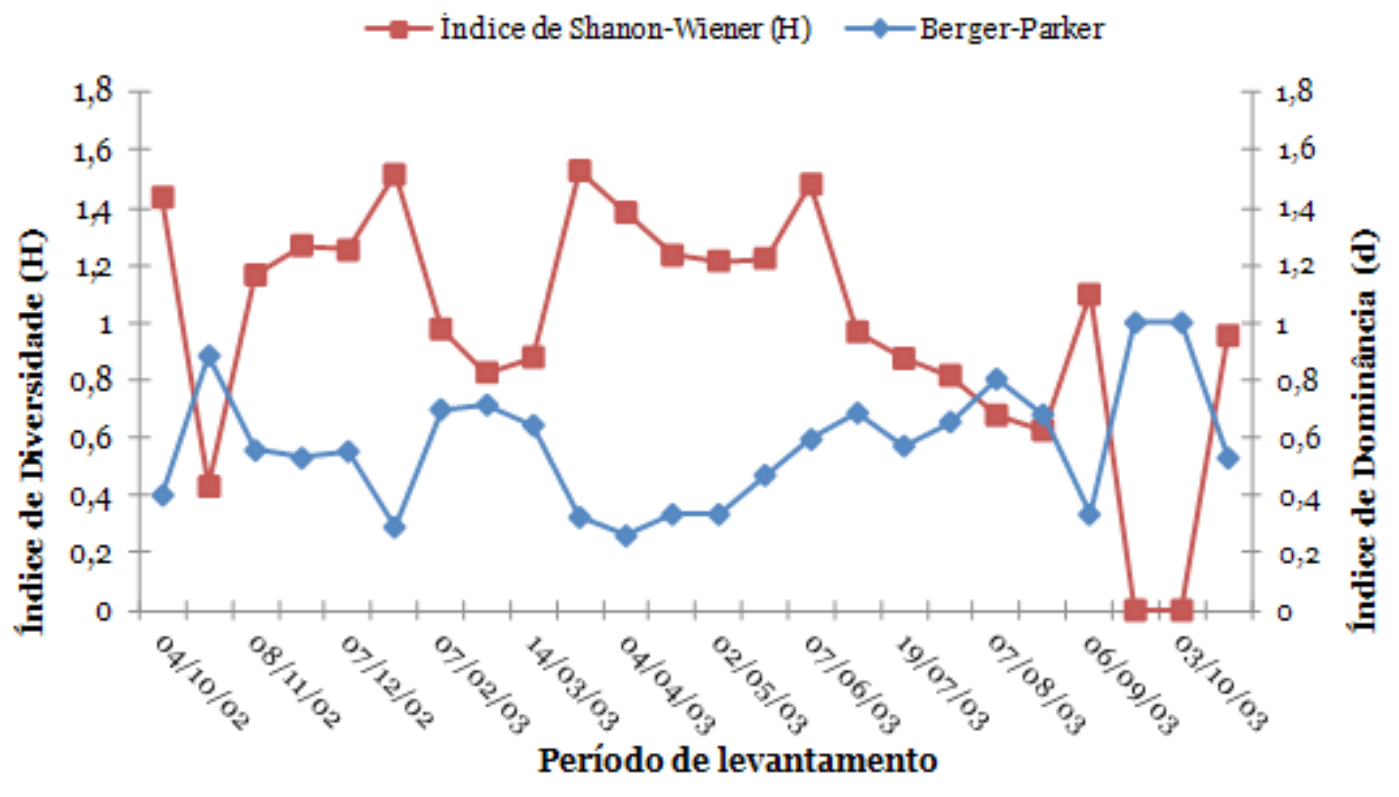

Figura 2. Relação entre o índice de diversidade de Shanon-Wiener (H’) e a dominância de Berger-Parker (d), entre formicídeos que atendem Aleurothrixus floccosus, associados à tangerina cv Poncã, cultivada em sistema orgânico, no período diurno. Seropédica, RJ, Fazendinha Agroecológica. Outubro/2002 - Outubro/2003.

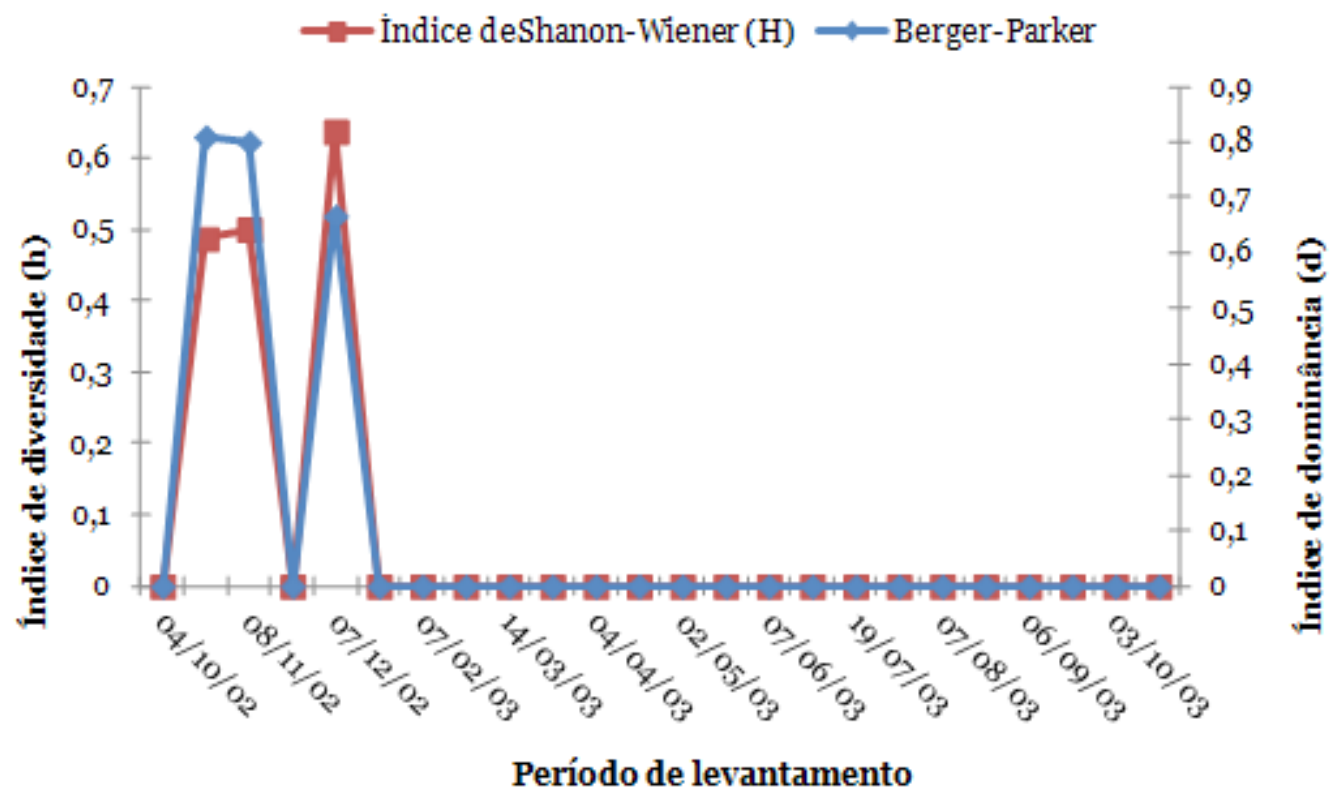

Figura 3. Relação entre o índice de diversidade de Shanon-Wiener (H’) e a dominância de Berger-Parker (d), entre formicídeos que atendem Aleurotachelus cruzi, associados à tangerina cv Poncã, cultivada em sistema orgânico, no período diurno. Seropédica, RJ, Fazendinha Agroecológica. Outubro/2002 - Outubro/2003. 
(mais de seis indivíduos, geralmente), e em Camponotus sp. e Camponotus rufipes (um ou dois indivíduos geralmente).

Constância e Frequência. As espécies de formigas mais constantes, associadas à A. floccosus, foram: Camponotus sp. (88\%), seguida por Brachymyrmex sp. e C. rufipes (75 e 71\%, respectivamente). As espécies com menor constância durante o período de levantamento foram: Crematogaster sp.2, E. brunneum e C. pusillus (4\% cada) (Tabela 2).

As espécies de formigas mais frequentes, em relação às demais foram Brachymyrmex sp. com 31,94\%, seguida por Crematogaster sp. 1 (27\%), sendo que as menos frequentes foram E. brunneum (0,22\%), Crematogaster sp.2 (o,44\%) e Dorymyrmex sp. (0,55\%).

Todas as espécies de formigas associadas a $A$. cruzi foram classificadas como acidentais. Entretanto, Crematogaster sp. 1 obteve um valor de constância de 21\%, ou seja, próximo a $25 \%$, o que configuraria uma espécie acessória, sendo esta a espécie mais frequente entre as demais (6o,98\%) (Tabela 3), reforçando desta forma a espécie é dominante.

As espécies associadas a $P$. bondari foram classificadas como acidentais, pois somente um indivíduo de cada espécie foi verificado (C. rufipes, C. atricefs e C. pusillus).

Em estudos de comunidades de formicídeos em pomares de cítricos, Castro et al. (1989) verificaram que Myrmicinae foi responsável por 50\% das 94 espécies coletadas e $66 \%$ dos indivíduos obtidos, destacando-se os gêneros Crematogaster, Solenopsis e Pheidole. A subfamília Formicinae foi encontrada igualmente de forma abundante, representando $26 \%$ dos indivíduos coletados e $22 \%$ das morfoespécies, com uma espécie entre as mais abundantes.

Os resultados do presente estudo demonstram que espécies dealeirodídeos ao receberem atendimento deformicídeos podem também estar recebendo a proteção destes, o que é preocupante, pois além do dificultar o controle, os aleirodídeos são associados à transmissão de vírus (KARASEV 2000).

O entendimento da proteção de aleirodídeos por formigas é de suma importância, principalmente em sistemas de cultivos orgânicos, pois a aplicação de defensivos agrícolas de origem sintética não é realizada e a partir deste entendimento melhores decisões e estratégias de controle poderão ser adotadas, visando minimizar os efeitos da ação dos aleirodídeos e o equilíbrio biológico, que é um dos princípios do Sistema Integrado de Produção Agroecológica (SIPA).

$\mathrm{O}$ alto grau de infestação verificado na população de A. floccosus e a associação com 12 espécies de formicídeos demonstram que há uma interação de atendimento mais estreita, quando comparada com as demais espécies de aleirodídeos e também que a produção de honeydew por esta espécie, pode favorecer uma maior proteção e dispersão por parte das formigas, podendo dificultar os programas de controle que venham a ser adotados.

Tabela 2. Constância e frequência das formigas atendentes de Aleurothrixus floccosus associadas à tangerina cv Poncã, cultivada em sistema orgânico, no período diurno. Seropédica, RJ, Fazendinha Agroecológica. Outubro/2002 - Outubro/2003.

\begin{tabular}{lcc}
\hline \multicolumn{1}{c}{ Formigas } & Constância $^{\mathbf{1}}$ & Frequência $^{\mathbf{2}}$ \\
\hline Brachymyrmex sp. & $\mathrm{W}$ & 0,3194 \\
C. rufipes & $\mathrm{w}$ & 0,1164 \\
C. atricefs & $\mathrm{z}$ & 0,0121 \\
C. crassus & $\mathrm{w}$ & 0,0538 \\
Camponotus sp. & $\mathrm{W}$ & 0,1811 \\
C. pusillus & $\mathrm{z}$ & 0,0132 \\
Crematogaster sp. 1 & $\mathrm{w}$ & 0,2700 \\
Crematogaster sp. 2 & $\mathrm{z}$ & 0,0044 \\
E. brunneum & $\mathrm{z}$ & 0,0022 \\
Pheidole sp. & $\mathrm{z}$ & 0,0121 \\
Dorymyrmex sp & $\mathrm{z}$ & 0,0055 \\
P. termitarius & $\mathrm{z}$ & 0,0099 \\
\hline
\end{tabular}

${ }^{1} \mathrm{~W}$ - espécies constantes; $\mathrm{y}$ - espécies acessórias; $\mathrm{z}$ - espécies acidentais.

${ }^{2}$ Frequência relativa ao número de indivíduos de cada espécie e o número total de indivíduos observados.

Tabela 3. Constância e frequência das formigas atendentes de Aleurotrachelus cruzi associadas à tangerina cv Poncã, cultivada em sistema orgânico, no período diurno. Seropédica, RJ, Fazendinha Agroecológica. Outubro/2002 - Outubro/2003.

\begin{tabular}{lcc}
\hline \multicolumn{1}{c}{ Formigas } & Constância $^{\mathbf{1}}$ & Frequência $^{\mathbf{2}}$ \\
\hline C. rufipes & $\mathrm{z}$ & 0,0244 \\
C. crassus & $\mathrm{z}$ & 0,0976 \\
Camponotus sp. & $\mathrm{z}$ & 0,2683 \\
Crematogaster sp. 1 & $\mathrm{z}$ & 0,6098 \\
\hline
\end{tabular}

${ }^{1} \mathrm{~W}$ - espécies constantes; y - espécies acessórias; $\mathrm{z}$ - espécies acidentais.

${ }^{2}$ Frequência relativa ao número de indivíduos de cada espécie e o número total de indivíduos observados.

\section{AGRADECIMENTOS}

Os autores agradecem ao Prof. R. Silva-Filho pela valiosa revisão e sugestões para a elaboração deste trabalho, e a todos os pesquisadores e funcionários da Fazendinha Agroecológica, em especial ao Dr. Raul Lucena D. Ribeiro (Prof. da UFRuralRJ) pelo apoio incondicional na realização dos trabalhos de campo.

\section{REFERÊNCIAS}

Addicott, J.F., 1978. Competition for mutualists: aphids and ants. Canadian Journal Zoology, 56: 2093-2096.

Brower, J. E., J.H. Zar \& C.N. von Ende, 1997. Field and laboratory methods for general ecology. WCB/ McGraw-Hill, $4^{\text {th }}, 273 \mathrm{p}$.

Buckley, R., 1987. Ant-Plant-Homopteran interactions. Advanced
Ecolology Research, 16: 53-85.

Carroll, C.R. \& D.H. Janzen, 1973. Ecology of foraging by ants. Annual Review of Ecology and Systematics, 4: 231-257.

Cassino, P.C.R. \& F.N. Nascimento, 1999. Aleirodídeos (Homoptera: Aleyrodidae) em plantas cítricas no Brasil: distribuição e identificação. Anais da Sociedade Entomológica do Brasil, 28: 75-83.

Cassino, P.C.R. \& W.C. Rodrigues, 2004. Monitoramento de insetos fitófagos, ácaros e inimigos naturais, p. 149-157. In: P.C.R. Cassino \& W.C. Rodrigues (Coords.), Citricultura Fluminense: principais pragas e seus inimigos naturais. Seropédica, EDUR, 168p.

Cassino, P.C.R., W.C. Rodrigues \& S.S.P. Souza, 2004. Aleirodídeos, p. 15-26. In: P.C.R. Cassino \& W.C. Rodrigues 
(Coords.), Citricultura Fluminense: principais pragas e seus inimigos naturais. Seropédica, EDUR, 168p.

Castro, A.G., M.V.B. Queiroz \& L.M. Araújo, 1989. Estrutura e diversidade de comunidades de formigas em pomar de citros. Anais da Sociedade Entomológica do Brasil, 18: 229-246.

Delabie, J.H.C., 2001. Trophobiosis between Formicidae and Hemiptera (Sternorrhyncha and Auchenorrhyncha): and overview. Neotropical Entomology, 30: 501-516.

Del-Claro K. \& P.S. Oliveira, 1996. Honeydew flicking by treehoppers provides cues to potential tending ants. Animal Behavior, 51: 1071-1075.

Fischer, M.K., W. Völkl, R. Schopf \& K.H. Hoffmann, 2002. Agespecific patterns in honeydew production and honeydew composition in the aphid Metopeurum fuscovirides: implications for ant-attendance. Journal Insect Physiology, 48: 319-326.

Fowler, H.G., 1988. Organização e regulação social em formigas. Etologia, 5: 235-247.

Fowler, H.G., L.C. Forti, C.R.F. Brandão, J.H.C. Delabie \& H.L Vasconcelos, 1991. Ecologia nutricional de formigas, p. 131223. In: A.R. Panizzi \& J.R.P. Parra (eds.) Ecologia Nutricional de insetos e suas implicações no manejo de pragas. São Paulo, Manole, 359p.

Gulan, P.J. \& M. Kosztarab, 1997. Adaptations in scale insects. Annual Review of Entomology, 42: 23-50.

Hendrix, D.L, Y. Wey \& J.E. Leggett, 1999. Homopteran honeydew sugar composition is determined by both the insect and plant species. Comparative Biochemical Physiology, 101B: 23-27.

Hölldobler, B. \& E.O. Wilson, 1990. The Ants. The Bleknap Press of the Harvard University Press. Cambridge Massachusetts, 732p.

Karasev, A.V., 2000. Genetic Diversity and evolution of closteroviruses. Annual Review of Phytopatology, 38: 293324.

Kelin, R.W., D. Kovac, A. Schellerich, \& U. Machwitz, 1992 Mealybug-carring by swarming queens of a Southheast Asain bamboo-inhabiting ant. Naturwissenschaften, 79: 422-423.

\section{Como citar este artigo:}

Rodrigues, W.C. \& P.C.R. Cassino, 2011. Interação entre Formigas e Aleirodídeos (Sternorrhyncha, Aleyrodidae) em Cultivo Orgânico de Tangerina cv. Poncã (Citrus reticulata Blanco). EntomoBrasilis, 4(3): 119-124. http://www.periodico.ebras.bio.br/ojs
Oliveira, P.S. \& C.R.F. Brandão 1991. The ant community associated with extrafloral nectaries in the Brazilian cerrados, p. 198-212. In: D.F. Cutler \& C. R. Huxley (Editors). Ant-Plant Interactions. Oxford University Press. Oxford.

Queiroz, J.M. \& P.S Oliveira, 2001. Tending ants protect honeydew-producing whiteflies (Homoptera: Aleyrodidae). Environmental Entomology, 30: 295-297.

Rodrigues, W.C., 2004. Homópteros (Homoptera: Sternorrhyncha) Associados à Tangerina CV. Poncã (Citrus reticulata Blanco) em Cultivo Orgânico e a Interação com Predadores e Formigas). Tese (Doutorado em Agronomia: Fitotecnia) - Universidade Federal Rural do Rio de Janeiro. 63p.

Rodrigues, W.C., 2005. DivEs - Diversidade de espécies. Versão 2.o. Software e Guia do Usuário. Disponível em: $<$ http://www.ebras.bio.br > (02 novembro 2005).

Samways, M.J, M. Nel \& A.J. Prins, 1982. Ants (Hymenoptera; Formicidae) foraging in citrus trees and attending honeydewproducing Homoptera. Phytophylactica, 14: 155-157.

Samways, M.J., 1983. Communitystructure ofants(Hymenoptera: Formicidae) in a series of habitats associated with citrus. Journal Applied Ecology, 20: 833-847.

Sun Microsystem, 2005. Open Office.org, versão 2.0 (BR): Sun Microsystem Inc. Conjunto de programas. Disponível em: $<$ www.openoffice.org $>$.

Way, M.J., 1963. Mutualism between ants and honeydewproducing Homoptera. Annual Review of Entomology, 8: 307-344.

Recebido em: 29/07/2011

Aceito em: 07/10/2011
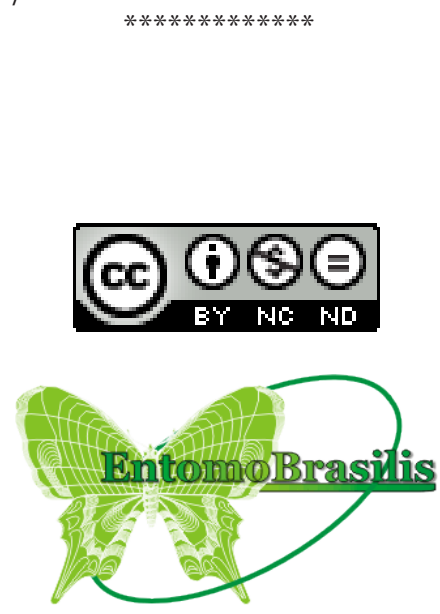

Aponte a câmera do celular, que possua leitor de QRCode, para acessar o artigo

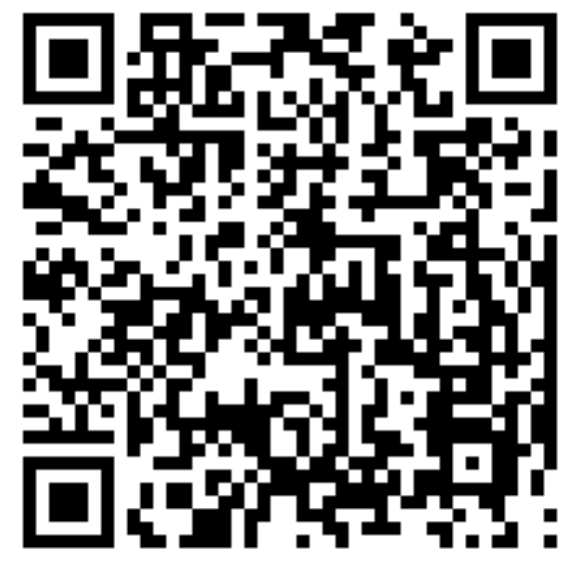

